TRANSACTIONS OF THE

AMERICAN MATHEMATICAL SOCIETY

Volume 218, 1976

\title{
REVERSIBLE DIFFEOMORPHISMS AND FLOWS
}

BY

\author{
ROBERT L. DEVANEY
}

\begin{abstract}
We generalize the classical notion of reversibility of a mechanical system. The generic qualitative properties of symmetric orbits of such systems are studied using transversality theory. In particular, we prove analogues of the closed orbit, Liapounov, and homoclinic orbit theorems for $R$-reversible systems.
\end{abstract}

0. Introduction. In classical terminology, a reversible mechanical system is one whose Hamiltonian assumes the particularly simple form $K+V$. Here $K$ is the kinetic energy of the system, and $V$ is the potential energy. One of the aims of this paper is to generalize this notion of reversibility. We then study the qualitative properties of such generalized systems from a more geometric point of view.

The first three sections of this paper give motivation for our definition of $R$-reversible vector fields and diffeomorphisms. In $\S 1$ we discuss the classical reversible systems in some detail. We show here that the classical definition of reversibility is equivalent to the existence of a $C^{\infty}$ involution $R$ of phase space having two special properties. First, the fixed point set of $R$ is an $n$-dimensional submanifold of the $2 n$-dimensional phase space, and second, $R$ "reverses" the Hamiltonian vector field, i.e., $T R\left(X_{H}\right)=-X_{H}$.

The next two sections deal with two classical Hamiltonian systems, the Störmer and the restricted three body problems. We prove here the existence of another $C^{\infty}$ involution $R_{1}$ of phase space having the same properties as above. Thus, in our terminology, these systems are examples of $R_{1}$-reversible systems.

An important reason for the choice of the Störmer and the restricted three body problems as examples is the fact that, for certain values of the energy, both systems admit cross-sections. These are codimension one submanifolds of the energy surface having the property that each orbit of the vector field intersects the submanifold at least once in every sufficiently large time interval. Hence the study of the qualitative properties of the vector field reduces to the study of the qualitative properties of the induced "first return" diffeomorphism of the section. Because of the $R$-reversibility of the Störmer and the restricted three body problems, these induced diffeomorphisms have special properties. Both can be written as a composition of two involutions $U \circ R$. Moreover, the fixed point sets of

Received by the editors November 6, 1974 and, in revised form, February 24, 1975. AMS (MOS) subject classifications (1970). Primary 58F05, $70 \mathrm{H} 99$. 
both $U$ and $R$ are submanifolds of the section having dimension equal to exactly one-half the dimension of the section. We call such diffeomorphisms $R$-reversible.

One interesting feature of these cross-sections is the fact that they are both submanifolds-with-boundary of the energy surfaces. The flow is everywhere tangent to the boundary and transverse to the interior of the section. Thus we have a somewhat different situation than what is usually meant by a cross-section for a flow. And so, in an appendix, we define and discuss the basic properties of these sections with boundary.

In the main part of this paper, we study the qualitative properties of $R$-reversible systems. More precisely, we consider a $2 n$-dimensional manifold $M$ together with a diffeomorphism $R$ of $M$ satisfying

(i) $R^{2}=$ identity,

(ii) $\operatorname{dim} \operatorname{Fix}(R)=n=1 / 2 \operatorname{dim} M$.

$R$ is called a reversing involution. Note that we do not require $M$ to be a symplectic manifold.

A smooth vector field $X$ on $M$, is then called $R$-reversible if $T R(X)=-X \circ R$.

Of primary importance in the study of such systems are the symmetric periodic orbits. These are closed orbits of $X$ which intersect Fix $(R)$ in exactly two points. The problem of finding such orbits is thus equivalent to the more geometric problem of finding self-intersections of the fixed point set of $R$ under the flow map. We remark that Birkhoff and De Vogelaere have used this technique to classify the symmetric periodic orbits in the restricted three body and the Störmer problems [3], [4], [8].

In $\S \S 6$ and 7 , we discuss the generic qualitative properties of $R$-reversible systems. In particular, Theorems 6.5 and 7.6 give Kupka-Smale theorems for $R$ reversible diffeomorphisms and vector fields.

Of interest here is the fact that reversible systems, near symmetric periodic orbits, behave qualitatively just like Hamiltonian systems. For example, Proposition 7.3 shows that, generically, symmetric periodic orbits lie on one-parameter families of closed orbits. Also, in $\S 8$, we discuss a Liapounov theorem for $R$-reversible systems. Theorem 8.1 shows that, near an elementary symmetric critical point with a purely imaginary characteristic exponent, there exists a one-parameter family of symmetric closed orbits converging to the critical point.

Both of these results are well known in the Hamiltonian case. The proofs here, however, are more geometric in the sense that they involve finding transversal intersections of two codimension $n$ submanifolds of $M$.

Similar methods yield a homoclinic orbit theorem in $\S 9$. We show that hyperbolic critical points of $R$-reversible vector fields may admit a stable homoclinic orbit, i.e. an orbit which is doubly asymptotic to the equilibrium and which, moreover, cannot be perturbed away. Under mild transversality assumptions, 
we also show that such a homoclinic orbit is a limit of a one-parameter family of symmetric closed orbits whose periods tend to infinity.

We remark that a similar theorem is known in the Hamiltonian case only for systems with two degrees of freedom [21].

The author would like to acknowledge the advice and encouragement of $\mathrm{C}$. Simon, A. Weinstein, and especially his advisor, S. Smale, during the time this paper was being written.

I. Reversible mechanical systems. In classical mechanics, a reversible mechanical system is a triple $(M, K, V)$ where $M$ is a smooth $n$-dimensional Riemannian manifold and $K, V$ are smooth real-valued functions on the cotangent bundle $T^{*} M$ of $M$. $K$, the kinetic energy, is given by $1 / 2\left|\alpha_{p}\right|^{2}$ where $\alpha_{p} \in T_{p}^{*} M$ and $|\cdot|$ is given by the Riemannian metric on $M$. The potential energy $V$ is constant on the fibers of $T^{*} M$ and hence may be considered as a real-valued function on the configuration space $M$.

Let $H=K+V . H$ is called a Hamiltonian for the system. Let $\Omega$ be the canonical symplectic form on $T^{*} M$. Then $H$ defines a vector field on $T^{*} M$ via the equation

$$
i_{X_{H}} \Omega=-d H .
$$

$X_{H}$ is called the Hamiltonian vector field associated with the system. Writing the coordinate expression for $X_{H}$ in local coordinates on the cotangent bundle gives the classical formulation of Hamilton's equations.

Such systems are called reversible for the following reason. Let $(q(t), p(t))$ be an integral curve of $X_{H}$, where $q$ is a coordinate on configuration space and $p$ represents the conjugate momenta. Then the local coordinate expression of Hamilton's equations give immediately that $(q(-t),-p(-t))$ is also a solution curve for the system. In other words, following configuration space trajectories of the system in the reverse direction also gives solutions of the system.

Now let $R: T^{*} M \rightarrow T^{*} M$ be given by $R\left(\alpha_{p}\right)=-\alpha_{p}$. We call $R$ a reversing involution of phase space. Note that $R^{*} \Omega=-\Omega$ so that $R$ is antisymplectic. The fixed point set of $R$ is the zero section of $T^{*} M$, and thus is a submanifold of $T^{*} M$ having dimension equal to one-half the dimension of $T^{*} M$.

The following proposition follows from the local coordinate expression of Hamilton's equations.

Proposition 1.1. Let $(M, K, V)$ be a reversible mechanical system and let $R$ be the reversing involution defined above. Then

(i) $H \circ R=H$,

(ii) $\operatorname{TR}\left(X_{H}\right)=-X_{H} \circ R$,

(iii) $\phi_{t} R=R \phi_{-t}$ where $\phi_{t}$ is the flow of $X_{H}$ on $T^{*} M$.

Note that (iii) above says that $\left(\phi_{t} R\right)^{2}=$ identity on $T^{*} M$; hence $\phi_{t} R=U_{t}$ is an involution. Let $F(R)$ and $F\left(U_{t}\right)$ be the fixed point sets of $R$ and $U_{t}$ respec- 
tively. Since $F(R)$ is the zero section of $T^{*} M$, it follows that $F\left(U_{t}\right)$ is also an $n$-dimensional submanifold of $T^{*} M$.

The intersections of $F(R)$ and $F\left(U_{t}\right)$ have special significance. If $p \in F(R)$ $\cap F\left(U_{t}\right)$, the orbit through $p$ is mapped onto itself by $R$ with a change of direction. Such an orbit is called symmetric. If an orbit is both symmetric and periodic, it is easy to see that the orbit intersects $F(R)$ exactly twice in any prime period. These intersections belong to $F\left(U_{\tau}\right) \cap F(R)$ and $F\left(U_{\tau / 2}\right) \cap F(R)$ where $\tau$ is the prime period of the orbit. They are points of symmetric conjunction. Such points have often played an important role in the discovery of periodic orbits. See, for instance, [3] and [6].

More generally, one can consider Hamiltonians of the form $H=K+P_{Y}+V$. Here $K$ and $V$ are the usual kinetic and potential energies, and $Y$ is a smooth vector field on configuration space. Then $P_{Y}$ is defined by $P_{Y}\left(\alpha_{p}\right)=\alpha_{p}(Y) . P_{Y}$ is called a momentum function. Thus $X_{H}$ is reversible in the classical sense iff $Y$ is identically zero.

Now the Riemannian metric on $M$ converts vector fields to one-forms and vice versa. Let $\tilde{Y}$ be the one-form dual to $Y$ under this correspondence. Define $\hat{R}: T^{*} M$ $\rightarrow T^{*} M$ by $\hat{R}\left(\alpha_{p}\right)=-2 \widetilde{Y}-\alpha_{p} . \hat{R}$ is a $C^{\infty}$ involution of $T^{*} M$ whose fixed point set is an $n$-dimensional submanifold of phase space. Furthermore, $H \circ \hat{R}=H$. Now if the one-form $\widetilde{Y}$ is closed, then an easy computation shows that $\hat{R}^{*} \Omega=$ - $\Omega$. It follows immediately that $T \hat{R}\left(X_{H}\right)=-X_{H} \circ \hat{R}$. Thus $X_{H}$ is reversed by an involution very similar to the reversing involution of $K+V$ systems. We thus call such systems $\hat{R}$-reversible.

II. The Störmer problem. In this section, we discuss an example of a classically reversible Hamiltonian system which also admits another involution which reverses the vector field.

Suppose a magnetic dipole is fixed at the origin in $R^{3}$ with magnetic moment pointing in the direction of the negative $z$-axis. The problem is to find the trajectories of a charged particle in this magnetic field. Interpreting the field as the earth's magnetic field, we get the setting of the classical Störmer problem. For more details, see [5], [6], [18], [20].

After several standard reductions [5], the problem reduces to a simple Hamiltonian system with two degrees of freedom. The configuration space is $M=$ $R^{2}-0$ and the phase space is the cotangent bundle $T^{*} M$. Let $(q, p)$ be coordinates on $T^{*} M$. Then the Hamiltonian is

where

$$
2 H(q, p)=|p|^{2}+V(q)
$$

$$
V\left(q_{1}, q_{2}\right)=\left(1 / q_{1}-q_{1} /|q|^{3}\right)^{2}
$$

Thus $H$ is of the form $K+V$ as in $\S 1$, and so $X_{H}$ is a classically reversible system. 
We wish to show, however, that $X_{H}$ may be considered $R_{1}$-reversible for a different reversing involution $R_{1}$. To see this, define

$$
R_{1}\left(q_{1}, q_{2}, p_{1}, p_{2}\right)=\left(q_{1},-q_{2},-p_{1}, p_{2}\right) \text {. }
$$

Then $R_{1}$ is antisymplectic and preserves the Hamiltonian. Moreover the plane $q_{2}=0, p_{1}=0$ is the fixed point set of $R_{1}$. We may thus consider $R_{1}$ as a reversing involution on $T^{*} M$. An easy computation then shows that $X_{H}$ is $R_{1}$-reversible.

Let $V_{e}=\tau^{*}\left(H^{-1}(e)\right)$, where $\tau^{*}: T^{*} M \rightarrow T^{*} M$ is the usual projection. For $e<1 / 32$, it is known that $V_{e}$ has two components, one bounded and one un. bounded. See Figure 1. Let $A_{e}$ denote the intersection of the bounded component of $V_{e}$ and the $x$-axis. $\bar{\Sigma}_{e}=\left(\tau^{*}\right)^{-1}\left(A_{e}\right)$ is a two sphere which is $R_{1}$-invariant. Furthermore, it is easy to see that the equator $\left(q_{1}, 0, p_{1}, 0\right)$ of this sphere is a periodic orbit $\gamma$ of $X_{H}$. Now $\bar{\Sigma}_{e}-\gamma$ is a disjoint union of two two-dimensional disks. Let $\Sigma_{e}$ denote one of them together with $\gamma$. Then $\Sigma_{e}$ is $R_{1}$-invariant.

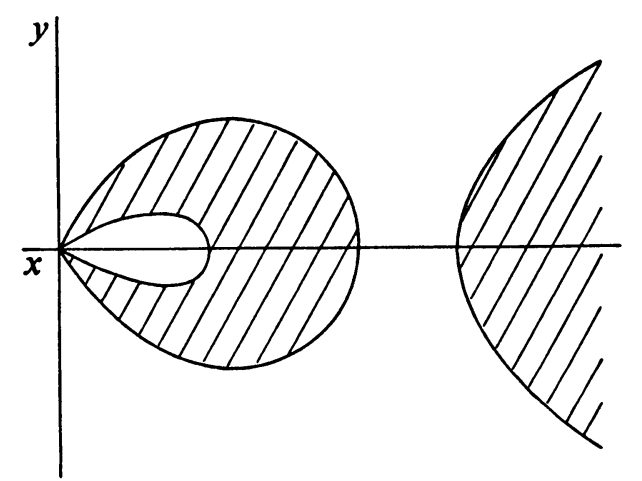

FIGURE 1. The shaded portion of the figure is the region $V_{e}$ for $e<1 / 32$ in the Störmer problem.

Concerning $\Sigma_{e}$ the following results are known. For proofs, see [8] and [7].

Proposition 2.1. (i) $\Sigma_{e}$ is a section with boundary for the vector field $X_{H}$ restricted to the energy surface $H^{-1}(e)$.

(ii) The first return map $\Phi: \Sigma_{e} \rightarrow \Sigma_{e}$ is a composition of two involutions, $\Phi=U \circ R_{1}$.

(iii) The fixed point sets of both $U$ and $R_{1}$ are one-dimensional submanifolds of $\Sigma_{e}$.

The definitions and basic properties of sections with boundary can be found in Appendix I.

III. The planar restricted three body problem. In this section we discuss an example of a classically irreversible Hamiltonian system, the restricted three body problem. We show here that there exists another involution of phase space 
that reverses the vector field, and thus that this system may also be considered $R_{1}$-reversible.

Using a rotating coordinate system, the configuration space is $M=R^{2}-$ $\{(-\mu, 0),(1-\mu, 0)\}$ and the phase space is $T^{*} M$ with the usual symplectic form $\Omega$.

The Hamiltonian is

$$
H=\frac{1}{2}|p|^{2}+q_{2} p_{1}-q_{1} p_{2}-\frac{\mu}{\rho(q)}-\frac{1-\mu}{\sigma(q)}-\frac{1}{2} \mu(1-\mu)
$$

where $(q, p) \in T^{*} M$ and

$$
\rho(q)=|q-(1-\mu, 0)|, \quad \sigma(q)=|q-(-\mu, 0)| .
$$

$C=-2 H$ is the well-known constant of Jacobi. For $C$ large, the region of motion is sketched in Figure 2. For a fixed value of $C$, let $N_{C}$ denote the component of the energy surface corresponding to motion near the larger mass. The vector field $X_{H}$ is everywhere tangent to $N_{C}$.

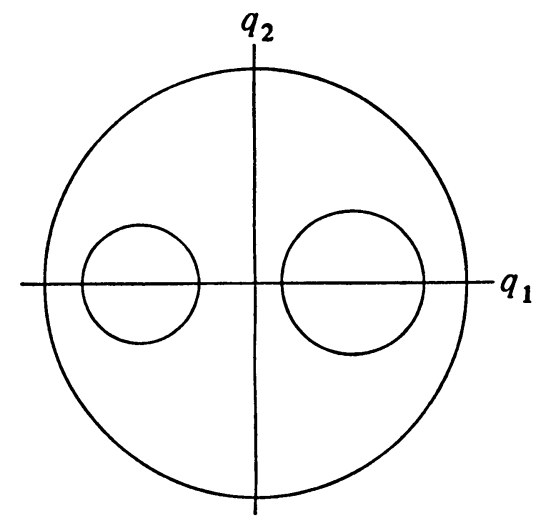

FIGURE 2. The region inside of the two smaller circles and outside of the largest circle is the region of motion for large values of the Jacobi constant in the restricted three body problem.

The Hamiltonian is of the form $K+L+V$, with $L \neq 0$. Hence the system is not classically reversible. However, there does exist an involution that reverses $X_{H}$.

Define $R_{1}: T^{*} M \rightarrow T^{*} M$ by

$$
R_{1}\left(q_{1}, q_{2}, p_{1}, p_{2}\right)=\left(q_{1},-q_{2},-p_{1}, p_{2}\right)
$$

as in the Störmer problem. As before, one checks easily that $T R_{1}\left(X_{H}\right)=-X_{H}{ }^{\circ} R$

We can now summarize the results of Poincaré and Birkhoff:

Proposition 3.1. For $C$ sufficiently large and $\mu$ sufficiently small:

(i) there exists an $R_{1}$-invariant section with boundary $\Sigma_{C}$ for $X_{H}$ on $N_{C}$. $\Sigma_{C}$ is diffeomorphic to an annulus. 
(ii) The first return map $L$ is a composition of two involutions $L=U R_{1}$.

(iii) The fixed point sets of both $U$ and $R_{1}$ are one-dimensional submanifolds of $\Sigma_{C}$.

For a proof, see Birkhoff [3].

The almost identical statements of Propositions 2.1 and 3.1 suggest a generalization of the notion of a reversible system. Below we define $R$-reversible diffeomorphisms and flows. The Störmer problem and the restricted three body problem then become specific examples of $R$-reversible vector fields. And their first return maps on the associated sections with boundary are examples of $R$-reversible diffeomorphisms.

IV. $R$-reversible diffeomorphisms and flows. Motivated by the previous examples, we now proceed to generalize the notion of reversibility.

Throughout, let $M$ be a compact, $C^{\infty}, 2 n$-dimensional manifold. Let Inv $^{s}(M)$ be the set of all $C^{s}$ involutions of $M$, i.e. all $C^{s}$ diffeomorphisms $f$ satisfying $f^{2}=$ identity. Since $\operatorname{Inv}(M)$ is the inverse image of the identity under the squaring map of $\operatorname{Diff}^{s}(M)$, it follows that $\operatorname{Inv}^{s}(M)$ has the Baire property. Let $I^{s}(M)$ be the subset of $\operatorname{Inv}^{s}(M)$ consisting of involutions whose fixed point set is an $n$-dimensional submanifold of $M$, i.e. $U \in I^{s}(M)$ iff $\operatorname{dim} F(U)=n=1 / 2 \operatorname{dim} M$ where $F(U)=$ fixed point set of $U$.

Fix a $C^{\infty}$ involution $R$ with $\operatorname{dim} F(R)=n . \quad L \in \operatorname{Diff}^{s}(M)$ is called $R$-reversible if $L=U R$, where $U \in I^{s}(M)$. Also, $X \in \Gamma^{s}(M)$ is $R$-reversible if $\operatorname{TR}(X)=-X$.

Let $\operatorname{Diff}_{R}^{s}(M)$ and $\Gamma_{R}^{s}(M)$ be the sets of all $C^{s} R$-reversible diffeomorphisms and flows respectively.

$\operatorname{Diff}_{R}^{s}(M)$ is the image of $I^{s}(M)$ under precomposition by $R$ and thus is a Baire space. $\Gamma_{R}^{s}(M)$ is a closed subspace of a Banach space. $\left\{X \in \Gamma^{s}(M) \mid \operatorname{TR}(X)=X\right\}$ is easily seen to be a closed complement of $\Gamma_{R}^{s}(M)$.

Let $L=U R \in \operatorname{Diff}_{R}^{s}(M)$. Set $U_{k}=L^{k} R$. $U_{k}$ is easily a $C^{s}$ involution of $M$. Let $F\left(U_{k}\right)=$ fixed point set of $U_{k}$. The following is easily proved:

Proposition 4.1. (i) $F\left(U_{k}\right)=L\left(F\left(U_{k-2}\right)\right)$,

(ii) $R F\left(U_{k}\right)=F\left(U_{-k}\right)$.

Hence $F\left(U_{2 k}\right)=L^{k} F(R)$ and $F\left(U_{2 k+1}\right)=L^{k} F(U)$. Thus $F\left(U_{k}\right)$ is an $n$-dimensional submanifold of $M$ for each $k$, and it follows that $L^{k}=U_{k} R \in$ $\operatorname{Diff}_{R}^{s}(M)$.

We call $F\left(U_{k}\right)$ the kth symmetry submanifold of $L$. The importance of these submanifolds stems from the following easy proposition.

Proposition 4.2. (i) If $j \neq k, F\left(U_{j}\right) \cap F\left(U_{k}\right) \subset \operatorname{per} L^{j-k}$.

(ii) Conversely, if $p \in F\left(U_{j}\right)$ is a periodic point of $L$, then $p \in F\left(U_{k}\right)$ for some $k \neq j$. 
Thus, certain periodic points of $L$ arise as intersections of the symmetry submanifolds. We call such points symmetric periodic points. Note that if one point on the orbit through $p$ is symmetric periodic, then all such points are.

A classification of symmetric periodic points is given by the following proposition. The proof is contained in De Vogelaere [6].

Proposition 4.3. Let $x$ be a symmetric periodic point of $L$. Then one of the iterates of $x$ belongs to

(i) $F\left(U_{2 k}\right) \cap F(R)$,

(ii) $F\left(U_{2 k+1}\right) \cap F(R)$,

(iii) $F\left(U_{2 k+1}\right) \cap F(U)$.

In particular, the subsets of these sets consisting of points with prime period $2 k$ (cases (i) and (iii)) or $2 k+1$ (case (ii)) are mutually exclusive and completely determine the set of symmetric periodic points.

Let $X \in \Gamma_{R}^{S}(M)$ have flow $\phi_{t}$. Then $\phi_{t} R=R \phi_{-t}$ for all $t$. Hence $U_{t}=\phi_{t} R$ is an involution of $M$ whose fixed point set is flow-isotopic to $F(R)$. Thus, $\phi_{t}=$ $U_{t} R$ is an $R$-reversible diffeomorphism for each $t$.

A critical element $\boldsymbol{\gamma}$ (singular point or closed orbit) of $X$ is called symmetric if $R(\gamma)=\gamma$. Note that all symmetric critical points must lie in $F(R)$, while all symmetric periodic orbits have exactly two intersections with $F(R)$ in any prime period.

The remaining critical elements are called nonsymmetric. They always occur in pairs $\gamma$ and $R(\gamma)$.

V. Linear reversible maps. Throughout this section, let $V$ denote a $2 n$ dimensional real vector space. Let $I(V)$ be the set of linear involutions of $V$ whose characteristic polynomial is $(x+1)^{n}(x-1)^{n}$. Fix $R \in I(V)$. A linear map $L$ is called $R$-reversible if $U=L R \in I(V)$. A linear map $A$ is called infinitesimally $R$-reversible if $A R=-R A$. The terminology is motivated by the Hamiltonian case.

Let $D_{R}(V)$ be the set of $R$-reversible linear maps and $d_{R}(V)$ the set of infinitesimally $R$-reversible linear maps. $2 n^{2}$

Proposition 5.1. (i) $D_{R}(V)$ is a $C^{\infty}$ submanifold of $\mathrm{GL}(V)$ of dimension

(ii) $T_{I} D_{R}(V)=d_{R}(V)$.

Proof. Define $\Phi: G L(V) \rightarrow \mathrm{GL}(V)$ by $\Phi(S)=S^{-1}$. The fixed point set of $\Phi$ is just the set of linear involutions of $V$, and thus is a $C^{\infty}$ submanifold of $\mathrm{GL}(V)$ by the Montgomery-Bochner theorem [10].

$I(V)$ is a connected component of the set of involutions; hence, $D_{R}(V)$ is 
is also a submanifold. The dimension of $D_{R}(V)$ follows by computing the +1 eigenspace of $T \Phi$.

For the second part of the proposition, if $\gamma_{t}$ is a smooth curve in $D_{R}(V)$ with $\gamma_{0}=I$, then $\gamma_{t}=\sigma_{t} R$ with $\sigma_{t} \in I(V)$ and $\sigma_{0}=R$. Since $\sigma_{t}^{2}=I$, we have $\dot{\sigma}_{0}=-R \dot{\sigma}_{0} R$. Hence $\dot{\gamma}_{0}=\dot{\sigma}_{0} R=-R \dot{\sigma}_{0} R R$ so that $\dot{\gamma}_{0} R=-R \dot{\gamma}_{0}$. Since $\operatorname{dim} d_{R}(V)=2 n^{2}$, it follows that $T_{I} D_{R}(V)=d_{R}(V)$.

Proposition 5.2. (i) Let $L \in D_{R}(V)$. If $\lambda$ is an eigenvalue of $L$ of multiplicity $k$, then $\lambda^{-1}$ is an eigenvalue of $L$ of multiplicity $k$. Furthermore, the eigenvalues +1 and -1 , if they occur, have even multiplicity.

(ii) Let $A \in d_{R}(V)$. If $\lambda$ is an eigenvalue of $A$ of multiplicity $k$, then $-\lambda$ is an eigenvalue of $A$ of multiplicity $k$. Furthermore, the eigenvalue 0 has even multiplicity if it occurs.

Proof. Let $L=U R \in D_{R}(V)$. Then $\operatorname{det}(U R-x I)=\operatorname{det}(U(U R-x I) U)=$ $\operatorname{det}(R U-x I)$ so the eigenvalues of $L$ and $L^{-1}$ are the same. $\operatorname{det} L=1$, so the eigenvalues -1 occur in pairs. Finally, $\operatorname{dim} V=2 n$, so the eigenvalues +1 also occur in pairs.

Part (ii) is similar.

The previous propositions suggest that $D_{R}(V)$ is contained in $\operatorname{SP}(2 n)$. However, if $n>1$, this is not the case, as the following example shows. Let

$$
U=\left|\begin{array}{rrrr}
1 & & & 0 \\
& -1 & & \\
& & 1 & \\
0 & & & -1
\end{array}\right|, R=\left|\begin{array}{rrrr}
1 & & & 0 \\
& 1 & & \\
& & -1 & \\
0 & & -1
\end{array}\right| \text {, }
$$

and $L=U R$. Then $L^{\tau} J L=-J$ where

$$
J=\left|\begin{array}{rr}
0 & I \\
-I & 0
\end{array}\right|
$$

so that $L$ is not symplectic.

Later, we will need the notion of a generic $R$-reversible linear map. Note that hyperbolicity is not a dense condition in $D_{R}(V)$ since an eigenvalue $\alpha+i \beta$ with $|\alpha+i \beta|=1$ and of multiplicity one is trapped on the unit circle and cannot be perturbed away. As in the symplectic case, we introduce the notion of an $N$ elementary $R$-reversible linear map.

Define the principal eigenvalues of $L \in D_{R}(V)$ to be the set of eigenvalues of modulus greater than one or of modulus one and imaginary part greater than zero. Take exactly half of the eigenvalues +1 and -1 . $L \in D_{R}(V)$ is $N$-elementary if the principal eigenvalues are multiplicatively independent over the integers between $-N$ and $N$, i.e. if $\prod_{i=1}^{n} p_{i}^{e_{i}}=1$ then $e_{i}=0$ for all $i$ where $p_{i}$ is a principal 
eigenvalue and $e_{i}$ is an integer, $\left|e_{i}\right|<N . L$ is elementary if $L$ is $N$-elementary for all $N$.

Note that $L$ elementary implies

(i) all of the principal eigenvalues are distinct,

(ii) none is a root of unity.

In a similar fashion, the principal eigenvalues of $A \in d_{R}(V)$ are those with real part greater than zero or real part zero and imaginary part greater than zero. Again, take exactly half of the eigenvalues 0 . Then $A$ is $N$-elementary if the principal eigenvalues $(\bmod 2 \pi i)$ are additively independent over the integers between $-N$ and $N$.

Let $D_{R}^{N}(V)\left(d_{R}^{N}(V)\right)$ be the set of $N$-elementary linear maps in $D_{R}(V)$ $\left(d_{R}(V)\right)$.

Proposition 5.3. $D_{R}^{N}(V)$ and $d_{R}^{N}(V)$ are open and dense in $D_{R}(V)$ and $d_{R}(V)$ respectively.

Proof. We show this for $d_{R}^{N}(V) ; D_{R}^{N}(V)$ is similar.

Fix $A \in d_{R}(V)$. We construct a recursive procedure for perturbing the principal eigenvalues.

Case 1. $A$ has an eigenvalue $\alpha+i \beta$ with $\alpha \neq 0$.

There exists $v_{1}, v_{2} \in V$ with $A v_{1}=\alpha v_{1}-\beta v_{2}$ and $A v_{2}=\beta v_{1}+\alpha v_{2}$. Let $W=\left[v_{1}, v_{2}, R v_{1}, R v_{2}\right]$. $\operatorname{dim} W=4$ since $\alpha \neq 0$. Define $\phi$ by

$$
\phi\left(v_{i}\right)=c v_{i}, \quad \phi\left(R v_{i}\right)=-c R v_{i},
$$

where $c$ is real. $\phi \in d_{R}(V)$ and the eigenvalues of $\phi+A$ on $\left[v_{1}, v_{2}\right]$ are $\pm((\alpha+c)+i \beta)$.

Case 2. $A$ has an eigenvalue i $\beta$ with $\beta=0$.

Pick $v_{1}, v_{2}$ as above. Note $R v_{1} \in\left[v_{1}, v_{2}\right]$ iff $R v_{2} \in\left[v_{1}, v_{2}\right]$. If $R v_{1}$ does not belong to $\left[v_{1}, v_{2}\right]$, Case 1 applies. If $R v_{1} \in\left[v_{1}, v_{2}\right]$, we may assume that $v_{1}, v_{2}$ are eigenvectors of $R$. So suppose $R v_{1}=v_{1}$ and $R v_{2}=-v_{2}$, and set $\phi v_{1}=c v_{2}$ and $\phi v_{2}=-c v_{1}$. Then $\phi \in d_{R}(V)$ and the eigenvalues of $\phi+A$ on $\left[v_{1}, v_{2}\right]$ are $\pm i(c+\beta)$.

Case 3. 0 is an eigenvalue of $A$.

Let $E^{0} A$ be the 0 -eigenspace of $A . E^{0} A$ is even dimensional and $R \in I\left(E^{0} A\right)$.

If $A v_{1}=0$ and $R v_{1}, v_{1}$ are linearly independent, proceed as in Case 1. So assume $R v_{1}=v_{1}\left(R v_{1}=-v_{1}\right.$ is similar $)$.

If there exists $v_{2}$ with $A v_{2}=v_{1}$, then there are two possibilities: $R v_{2} \in$ $\left[v_{1}, v_{2}\right]$ or $\operatorname{dim}\left[v_{1}, v_{2}, R v_{2}\right]=3$. In the first case, we may assume $R v_{2}=-v_{2}$ and

$$
A=\left|\begin{array}{ll}
0 & 1 \\
0 & 0
\end{array}\right|
$$


in the basis $v_{1}, v_{2}$. Set $v_{1}=c v_{2}$ and $v_{2}=-c v_{1}$ and perturb as in Case 2. In the second case, set

$$
\phi=\left|\begin{array}{rrr}
0 & 0 & 0 \\
0 & 0 & c \\
0 & -c & 0
\end{array}\right|
$$

in the basis $v_{1}, v_{2}, R v_{2} . \phi R=-R \phi$ and the eigenvalues of $A+\phi$ are $0, \pm i c$, so two of them have been perturbed away from zero.

A combination of such perturbations results in $A \mid E^{0} A$ is identically zero. Then the previous cases apply to make $A N$-elementary.

As a corollary, the set of elementary maps in $d_{R}(V)$ and $D_{R}(V)$ is residual, and hence dense.

For generic properties of $R$-reversible vector fields, we need some further properties of $D_{R}(V)$. Let $E^{\lambda} R$ denote the $\lambda$-eigenspace of $R$. Fix $v \in E^{-1} R$ and let $J_{v}=\left\{L \in D_{R} V \mid v \in E^{-1} U, L=U R\right\}$.

Any $L \in J_{v}$ has $v$ as a +1 eigenvector; hence one occurs as an eigenvalue of $L$ of multiplicity at least two. $L$ is called sub-N-elementary if the remaining $n-1$ principal eigenvalues are nontrivially multiplicatively independent over the integers between $-N$ and $N$.

Let $J_{v}^{N}$ denote the set of $L \in J_{v}$ that are not sub-N-elementary.

Proposition 5.4. (i) $J_{v}$ is a submanifold of $D_{R}(V)$ of codimension $n$.

(ii) $J_{v}^{N}$ is a finite union of submanifolds of $J_{v}$, each having codimension at least one in $J_{v}$.

Proof. Define $\pi: G L(V) \rightarrow I(V)$ by $\pi(g)=g R g^{-1} . \pi$ is a submersion. Also define $\Phi: \mathrm{GL}(V) \rightarrow E^{1}(R)$ by

$$
\Phi(g)=\left(R g^{-1}+g^{-1}\right)(v) .
$$

A computation shows that $\Phi$ is transverse to $0 \in E^{1} R$; hence $\Phi^{-1}(0)$ is a submanifold of GL(V) of codimension $n$. Now $g \in \Phi^{-1}(0)$ iff $g R g^{-1}(v)=-v$ iff $v \in E^{-1}\left(g R g^{-1}\right)$. Thus $\pi^{-1}(U) \in \Phi^{-1}(0)$ if $v \in E^{-1}(U)$. Since $\pi$ is a submersion, $\pi\left(\Phi^{-1}(0)\right)$ is a submanifold of $I(V)$ of codimension $n$ and (i) follows.

A method similar to Abraham-Robbin [2, p. 99] shows that $J_{v}^{N}$ is a finite union of submanifolds of $J_{v}$. The codimension of $J_{v}^{N}$ follows from the fact that sub- $N$-elementary $R$-reversible linear maps are dense in $J_{v}$ (proof as in 5.3).

VI. Generic $R$-reversible diffeomorphisms. In this section, we prove a Kupka-Smale theorem for $R$-reversible diffeomorphisms. As in the linear case, hyperbolicity is not a generic property of $\operatorname{Diff}_{R}^{s}(M)$, since, at symmetric periodic points, eigenvalues can be trapped on the unit circle. Thus we weaken the notion of hyperbolicity to obtain generic properties. 
Let $x$ be a symmetric periodic point of $L \in \operatorname{Diff}_{R}^{s}(M)$. Then $x$ is an elementary (resp. $N$-elementary) symmetric periodic point of $L$ of period $k$ if $T_{x} L^{k}$ is elementary (resp. $N$-elementary). If $x$ is any periodic point of $L$ of period $k$, $x$ is called generic (resp. $N$-generic) if either

(i) $x$ is an elementary ( $N$-elementary) symmetric periodic point of period $k$, or

(ii) $x$ is a hyperbolic nonsymmetric periodic point.

Let $G^{s}(k, N)$ be the set of all $C^{s} R$-reversible diffeomorphisms all of whose periodic points of period $\leqslant k$ are $N$-generic. Define $G^{s}(k)=\bigcap_{N=0}^{\infty} G^{s}(k, N)$ and $G^{s}=\bigcap_{k=1}^{\infty} G^{s}(k)$.

We need one more definition. $L \in \operatorname{Diff}_{R}^{s}(M)$ is called $k$-transversal if

(i) $k$ is even and $F\left(U_{k}\right)$ 历 $F(R), F\left(U_{k+1}\right)$ 历 $F(U)$,

(ii) $k$ is odd and $F\left(U_{k}\right)$ 币 $F(R)$.

Let $\Lambda^{s}(k)$ be the set of $k$-transversal $C^{s} R$-reversible diffeomorphisms.

The proof that $G^{s}(k)$ is residual requires the following four lemmas. The proofs use the notion of pseudotransversality. See Robinson [14] for the definitions and basic properties.

Lemma 6.1. Suppose $s \geqslant 1, k \geqslant 0$, and $G^{s}(2 k)$ is dense in $\operatorname{Diff}_{R}^{s}(M)$. Then $\left\{L \in \operatorname{Diff}_{R}^{s}(M) \mid F\left(U_{2 k+1}\right) \pitchfork F(R)\right\}$ is open and dense in $\operatorname{Diff}_{R}^{s}(M)$.

Proof. Consider the action of $\operatorname{Diff}^{s}(M)$ on $I^{s}(M)$ defined by $g \cdot U=g U g^{-1}$ where $g \in \operatorname{Diff}^{s}(M), U \in I^{s}(M)$. This action admits local cross-sections [11]. That is, given $U \in I^{s}(M)$, there is a neighborhood $O$ of $U$ in $I^{s}(M)$ and a continuous map $\chi: 0 \rightarrow \operatorname{Diff}^{s}(M)$ satisfying

$$
\chi(U)=1_{M}, \quad \chi(S) \cdot U=S .
$$

Fix $\bar{U} \in I^{s}(M)$. Let orb $(\bar{U})$ denote the orbit of $\bar{U}$ under this action. $\operatorname{orb}(\bar{U})$ is open in $I^{s}(M)$. Define $\pi: \operatorname{Diff}^{s}(M) \rightarrow \operatorname{orb}(\bar{U})$ by $\pi(g)=g \bar{U} g^{-1}$ and set $D_{k}=\left\{g \in \operatorname{Diff}^{s}(M) \mid\left(g \bar{U} g^{-1} R\right) \in G^{s}(k)\right\}$. Let $\rho^{k}: \operatorname{Diff}^{s}(M) \rightarrow C^{s}(F(\bar{U}), M)$ be given by

$$
\rho^{k}(g)(p)=\left(g \bar{U} g^{-1} R\right)^{k} g(p) .
$$

$\rho^{k}(g)(F(\bar{U}))=F\left(\left(g \bar{U} g^{-1}\right)_{2 k+1}\right)$ by Proposition 4.1. We show $\rho^{k}$ is $C^{s}$ pseudotransverse to $F(R)$.

Let $D_{2 k}$ be the dense set in $\operatorname{Diff}^{s}(M), \Gamma^{s}(M)$ the Banach space of perturbations, and $\Psi_{g}: \Gamma^{s}(M) \rightarrow \operatorname{Diff}^{s}(M)$ given by $\Psi_{g}(X)=\phi_{1} g$ where $\phi_{t}=$ flow of $X$ and $g \in D_{2 k}$. $\rho^{k}$ is a $C^{1}$ pseudorepresentation.

The result is clear if $k=0$. If $k>0$, set $U=g \bar{U} g^{-1}$ and $L=U R$. If $\rho^{k}(g)(p)=g(p)$, then $T_{g(p)} L^{k}$ is elementary for $g \in D_{2 k}$, so $T_{g(p)} L^{2 k}$ is elementary. It follows that $T_{g(p)} L^{k}\left(T_{g(p)} F(U)\right)$ contains a closed complement of $T_{g(p)} F(R)$. 
The second case is $p^{k}(g)(p)=q \in F(R)$, but $q \neq g(p)$. If $L^{i}(g(p))=q$ for $i<k, F\left(U_{2 k+1}\right)$ T $F(R)$ as above. If $L^{k}(g(p))=q$ but $L^{i}(g(p)) \neq q$ for $i<k$, take a neighborhood $N$ of $q$ with $U(N) \cap N=\varnothing$ and $L^{i}(N) \cap N=\varnothing$ for $1 \leqslant i$ $\leqslant k$. Let $X \in \Gamma^{s}(M)$ have support in $N$. Then a computation shows that

$$
T_{(0, p)}\left(e v \circ \rho^{k} \circ \Psi_{g}\right)\left(X, 0_{p}\right)=X_{p} .
$$

These two cases thus yield that

$$
e v \circ \rho^{k} \circ \Psi_{g} \Pi_{(0, M)} F(R),
$$

and it follows that $\left\{g \in \operatorname{Diff}^{s}(M) \mid\left(g \bar{U} g^{-1} R\right) g F(\bar{U}) 历 F(R)\right\}$ is open and dense in $\operatorname{Diff}^{s}(M)$. Since $\pi$ is an open map, $\left\{U \in \operatorname{orb}(\bar{U}) \mid F\left(U_{2 k+1}\right) 币 F(R)\right\}$ is also open and dense.

Since there exists at most a countable number of orbits of $\chi[11]$, the lemma follows.

LEMma 6.2. Suppose $s \geqslant 1, k \geqslant 1$, and $G^{s}(2 k-1)$ is dense in $\operatorname{Diff}_{R}^{s}(M)$. Then $\left\{L \in \operatorname{Diff}_{R}^{s}(M) \mid F\left(U_{2 k}\right)\right.$ 历 $\left.F(R)\right\}$ is open and dense in $\operatorname{Diff}_{R}^{s}(M)$.

Proof. Let $D_{2 k-1}$ be the dense set and define $\rho^{k}: \operatorname{Diff}^{s}(M) \rightarrow$ $C^{s}(F(R), M)$ by

$$
\rho^{k}(g)(p)=\left(g U g^{-1} R\right)^{k}(p) .
$$

$\rho^{k}$ is $C^{s}$ pseudotransverse to $F(R)$ as before. The lemma then follows.

LEMMA 6.3. Suppose $s \geqslant 1, k \geqslant 1$, and $G^{s}(2 k-1)$ is dense in $\operatorname{Diff}_{R}^{s}(M)$. Then $\left\{L \in \operatorname{Diff}_{R}^{s}(M) \mid F\left(U_{2 k+1}\right)\right.$ 历 $\left.F(R)\right\}$ is open and dense in $\operatorname{Diff}_{R}^{S}(M)$.

Proof. Since $R F\left(U_{k}\right)=F\left(U_{-k}\right)$, the previous lemmas give

$$
\left\{L \in \operatorname{Diff}_{R}^{s}(M) \mid F\left(U_{-2 k}\right) \text { 历 } F(R)\right\}
$$

is open and dense.

To see the proof of 6.3 , note $F\left(U_{2 k+1}\right)$ 历 $F(U)$ iff $\left\{\bar{U} g^{-1} R g\right)^{k} F(\bar{U})$ 历 $F(\bar{U})$ iff $\left(g^{-1} \operatorname{Rg} \bar{U}\right) F(\bar{U}) 历 F(\bar{U})$. Hence the result follows from 6.2 by interchanging the roles of $R$ and $U$.

As a consequence of these three lemmas, we have that if $s \geqslant 1$ and $G^{s}(k-1)$ is dense in $\operatorname{Diff}_{R}^{s}(M)$, then $\Lambda^{s}(k)$ is open and dense in $\operatorname{Diff}_{R}^{s}(M)$.

Now let $\widetilde{\Lambda}^{s}(k)$ be the set of $C^{s} R$-reversible diffeomorphisms satisfying $T_{x} L^{k}$ has no eigenvalue one at symmetric periodic points of prime period $k$.

Lemma 6.4. Let $s \geqslant 1$ and suppose $G^{s}(k-1)$ is dense in $\operatorname{Diff}_{R}^{s}(M)$. Then $\widetilde{\Lambda}^{s}(k)$ is dense and open in $\operatorname{Diff}_{R}^{s}(M)$.

Proof. Openness follows immediately from openness of $C^{S}$ diffeomorphisms that are transverse to the diagonal. 
For density, assume first that $L \in \Lambda^{s}(k)$ has $p \in F\left(U_{k}\right) \cap F(R)$ as a symmetric periodic point with prime period $k . F\left(U_{k}\right) 币 F(R)$ so $F\left(U_{k}\right) \cap F(R)$ is a finite set of points. Thus we may take a neighborhood $O$ of $p$ satisfying

(i) $F\left(U_{k}\right) \cap F(R) \cap 0=\{p\}$,

(ii) $L^{i}(0) \cap 0=\varnothing$ for $1 \leqslant i \leqslant k$.

Now the results of $\S 5$ give the existence of $A \in L\left(T_{p} M, T_{p} M\right)$ arbitrarily close to the identity so that $A \circ T_{p} U_{k} \circ A^{-1} \circ T_{p} R$ is elementary. Choose $X \in$ $\Gamma^{s}(M)$ with flow $\phi_{t}$ satisfying:

(i) supp $X \subset 0$,

(ii) $X(p)=0$,

(iii) $T_{p} \phi_{1}=A$,

(iv) the $C^{s}$ size of $X$ is small.

Define $\widetilde{U}=\phi_{1} U \phi_{-1}$ and $\tilde{L}=\tilde{U} R$. Then $\tilde{L}$ is arbitrarily close to $L$, $\tilde{L}^{k}(p)=p$, and $T_{p} \widetilde{L}^{k}=T_{p}\left(\phi_{1} U_{k} \phi_{-1} R\right)$ is elementary.

If $p \in F(U) \cap F\left(U_{k+1}\right)$, a similar procedure makes $p$ an elementary symmetric periodic point. By the classification of symmetric periodic points, this shows $\tilde{\Lambda}^{s}(k)$ is dense.

THEOREM 6.5. Let $M$ be a compact, 2 n-dimensional, $C^{\infty}$ manifold. If $s \geqslant 1, G^{s}(k)$ is residual in $\operatorname{Diff}_{R}^{s}(M)$. Thus, $G^{s}$ is also residual.

Proof. We show this by induction. Assume $G^{s}(k-1)$ is dense. Lemma 5.4 shows that $G^{s}(k-1)$ dense implies $\widetilde{\Lambda}^{s}(k)$ is open and dense in $\operatorname{Diff}_{R}^{s}(M)$.

Define $\rho: \operatorname{Diff}_{R}^{s}(M) \rightarrow C^{s}(M, M \times M)$ by $\rho(L)(x)=\left(x, L^{k}(x)\right)$. $\rho$ is a $C^{1}$ pseudorepresentation; we show $\rho$ is $C^{S}$ pseudotransverse to the diagonal $\Delta$ in $M \times M$.

Let $D_{k}=G^{s}(k-1) \cap \tilde{\Lambda}^{s}(k)$ be the dense set in $\operatorname{Diff}_{R}^{s}(M)$ and $\Gamma^{s}(M)$ the Banach space of perturbations at $L=U R \in D_{k}$. Define $\Psi_{L}: \Gamma^{S}(M) \rightarrow \operatorname{Diff}_{R}^{s}(M)$ by $\Psi_{L}(X)=\phi_{1} U \phi_{-1} R$ where $\phi_{t}=$ flow of $X$. ev॰ $\rho \circ \Psi_{L}$ is $C^{s}$ by the parametrized flow theorem [2, p. 57]. So we must show $e v \circ \rho \circ \Psi_{L} \Pi_{0, M} \Delta$.

Suppose $L^{k}(x)=x$. If $L^{i}(x)=x$ for $1 \leqslant i \leqslant k$, then $T_{x} L^{i}$ is generic, so $T_{x} L^{k}$ is generic and $e v \circ \rho \circ \Psi_{L} \Pi_{0, x} \Delta$. If $y$ is symmetric periodic of prime period $k, T_{y} L^{k}$ has no eigenvalue one, so $e v \circ \rho \circ \Psi_{L} \nwarrow_{0, y} \Delta$. Finally, if $x$ is nonsymmetric of prime period $k$, there exists a neighborhood $N$ of $x$ such that $R(N) \cap N=\varnothing$ and $L^{i}(N) \cap N=\varnothing$ for $1 \leqslant i \leqslant k$. Let $X \in \Gamma^{S}(M)$ have support in $N$. Then

$$
T_{(0, x)}\left(e v \circ \rho \circ \Psi_{L}\right)\left(X, 0_{p}\right)=X_{p}
$$

so $T\left(e v \circ \rho \circ \Psi_{L}\right)$ is surjective. Thus, $e v \circ \rho \circ \Psi_{L} \prod_{0, M} \Delta$.

It follows that $B_{k}=\left\{L \in \operatorname{Diff}_{R}^{s}(M) \mid \rho(L) 历 \Delta\right\}$ is open and dense in $\operatorname{Diff}_{R}^{s}(M)$. The proof of Lemma 6.4 then shows that $G^{s}(k)$ is dense in $B_{k} \cap$ $G^{s}(k-1)$. Using the fact that $G^{s}(k, N)$ is open in $\operatorname{Diff}_{R}^{s}(M)$, it follows that 
$G^{s}(k)$ is residual. This completes the proof of the theorem.

Note that, in the above proof, we cannot use the usual transversal-to-thediagonal argument to remove the eigenvalue one, since at symmetric periodic points, $T\left(e v \circ \rho \circ \Psi_{L}\right)$ spans only the -1 eigenspace of $T_{x} U_{k}$.

VII. Generic $R$-reversible flows. In this section, we prove a Kupka-Smale theorem for $R$-reversible vector fields. The proof is similar to Robinson [13]; hence we only sketch the necessary modifications.

Again, $M$ is a compact, $C^{\infty}, 2 n$-dimensional manifold, and $R$ is a fixed $C^{\infty}$ involution of $M$, with $\operatorname{dim} F(R)=n$.

Let $X \in \Gamma_{R}^{S}(M)$. $X$ has a symmetric critical point at $p \in M$ if $p \in F(R)$ and $X_{p}=0$. A symmetric critical point is called $N$-elementary if the Hessian of $X$ at $p, \dot{X}(p)$, is an $N$-elementary infinitesimally $R$-reversible map. Let $H^{s}(N)$ be the set of $X$ such that

(i) all symmetric critical points of $X$ are $N$-elementary,

(ii) all nonsymmetric critical points of $X$ are hyperbolic. Let $H^{s}=\bigcap_{N=1}^{\infty} H^{s}(N)$.

Lemma 7.1. Let $X \in \Gamma_{R}^{s}(M)$ have an isolated zero at $p \in F(R)$. If $s \geqslant 1$, there exists $X \in \Gamma_{R}^{S}(M)$ arbitrarily close to $X$ having $p$ as an elementary symmetric critical point.

Proof. Take $C^{\infty}$ coordinates $\left(x_{i}, y_{i}\right)$ on a neighborhood $N$ of $p$ satisfying

(i) $x_{i}(p)=0=y_{i}(p)$,

(ii) the local representative of $R$ is given by $\left(x_{i}, y_{i}\right) \rightarrow\left(x_{i},-y_{i}\right)$.

Condition (ii) is possible by the Montgomery-Bochner theorem [10].

Let $g$ be a $C^{S}$ small bump function with support in $N$ and $g=1$ on a neighborhood of $p$ in $N$. Let $Y_{1}=c g\left(x_{i}\left(\partial / \partial y_{j}\right)-y_{j}\left(\partial / \partial x_{i}\right)\right)$ where $c$ is real. $Y_{1} \in$ $\Gamma_{R}^{s}(M)$. Let $W_{i j} \subset T_{p}(M)$ be spanned by $\partial / \partial x_{i}$ and $\partial / \partial y_{j}$. Then

$$
\left.\dot{Y}_{1}(p)\right|_{w_{i j}}=\left(\begin{array}{cc}
0 & -c \\
c & 0
\end{array}\right)
$$

in this basis.

Also let $z_{i j}=x_{i}+y_{j}$ and $\bar{z}_{i j}=x_{i}-y_{j}$. Define

$$
Y_{2}=c g\left(z_{i j}\left(\partial / \partial z_{i j}\right)-\bar{z}_{i j}\left(\partial / \partial \bar{z}_{i j}\right)\right) \text {. }
$$

$Y_{2} \in \Gamma_{R}^{s}(M)$ and, in the basis $\partial / \partial z_{i j}, \partial / \partial \bar{z}_{i j}$

$$
\left.\dot{Y}_{2}(p)\right|_{w_{i j}}=\left(\begin{array}{cc}
c & 0 \\
0 & -c
\end{array}\right) .
$$

The proof of Proposition 5.3 shows that a combination of such perturbations makes $p$ an elementary symmetric critical point. 
THEOREM 7.2. If $s \geqslant 1, H^{s}(N)$ is open and dense in $\Gamma_{R}^{s}(M)$ and $H^{s}$ is residual.

Proof. First define $\rho: \Gamma_{R}^{s}(M) \rightarrow C^{s}(M, T M)$ by $\rho(X)=X_{p}$. Let $T F(R)$ denote the tangent bundle of $F(R)$ regarded as a $C^{\infty}$ embedded submanifold of TM. $\rho(X)(p) \in T F(R)$ iff $p \in F(R)$ and $X(p)=0$.

If $s \geqslant 1, e v \circ \rho$ is $C^{s}$ transverse to $T F(R)$. Hence

$$
\Lambda=\left\{X \in \Gamma_{R}^{s}(M) \mid X 历 T F(R)\right\}
$$

is open and dense in $\Gamma_{R}^{s}(M)$. If $X \in \Lambda$, the set of symmetric critical points of $X$ is finite. Lemma 7.1 applies to make $\Lambda_{N}=\left\{X \in \Gamma_{R}^{S}(M) \mid X\right.$ has only $N$-elementary symmetric critical points open and dense in $\Gamma_{R}^{S}(M)$.

Now $\rho$ is also $C^{s}$ pseudotransverse to $(T M)_{0}$, where $\Lambda_{N}$ is the dense set and $\Gamma_{R}^{S}(M)$ is the Banach space of perturbations. Arbitrary perturbations in a neighborhood of a nonsymmetric critical point make that point hyperbolic. Hence, $H^{s}(N)$ is open and dense in $\Gamma_{R}^{S}(M)$.

Let $X \in \Gamma_{R}^{S}(M)$ have $\gamma$ as a symmetric periodic orbit of period $2 \tau$ through $p \in F(R)$. Choose an $R$-invariant neighborhood $N$ of $p$ with coordinates $\left(x_{i}, y_{i}\right)$ satisfying

(i) the local representative of $R$ in $N$ is given by $\left(x_{i}, y_{i}\right) \rightarrow\left(x_{i},-y_{i}\right)$,

(ii) $X=\partial / \partial y_{n}$ on $N$.

Then $y_{n}=0$ defines an $R$-invariant local transversal section $\Sigma_{p}$ for $X$ at $p$. By the implicit function theorem, there exist neighborhoods $O$ of $X$ in $\Gamma_{R}^{s}(M)$ and $V$ of $p$ in $\Sigma_{p}$ and $C^{s}$ maps

$$
T: 0 \times V \rightarrow \mathbf{R}, \quad \Phi: 0 \times V \rightarrow \Sigma_{p}
$$

satisfying

$$
\Phi(Y, q)=\Phi_{T(Y, q)}^{Y}(q)
$$

where $\phi_{t}^{Y}=$ flow of $Y . \Phi$ is called a Poincare map. When dealing with symmetric periodic orbits, we always assume $\Sigma_{p}$ is $R$-invariant as above.

Since $\gamma$ is symmetric periodic of period $2 \tau, q=\phi_{\tau}(p) \in F(R)$. By choosing $O$ smaller if necessary, there exists an open neighborhood $W$ of $q$ in $F(R)$ and a $C^{S}$ map $\Psi: 0 \times W \rightarrow \Sigma_{p}$ satisfying $\Psi(Y, y)=x$ iff

$$
\left.\phi_{1 / 2 T(Y, \Phi}^{Y} \bar{Y}^{-1}(x)\right) \Phi_{Y}^{-1}(x)=y
$$

where $\Phi_{Y}(x)=\Phi(Y, x) . \Psi$ picks out those orbits which cross $W$ during the first iteration of the Poincaré map. $\Psi$ is called a semi-Poincaré map.

For fixed $Y \in 0$, the map $\Phi_{Y}$ is a $C^{s}$ diffeomorphism. Note that $\Phi_{Y}^{-1}(x)=$ $\left.\phi_{-T(Y, \Phi}^{Y} \bar{Y}^{1}(x)\right)(x)$. Hence 


$$
\begin{aligned}
\Phi_{Y}(R(x)) & =\phi_{T(Y, R(x))}^{Y}(R(x))=R \phi_{-T(Y, R(x))}^{Y}(x) \\
& =R \phi_{-T\left(Y, \Phi_{Y}^{Y}(x)\right)}^{Y}(x)=R \Phi_{Y}^{-1}(x)
\end{aligned}
$$

where reversibility gives $T(Y, R(x))=T\left(Y, \Phi_{Y}^{-1}(x)\right)$.

Thus we have $\left(\Phi_{Y} \circ R\right)^{2}=$ identity on a neighborhood of $p$ in $V$. Hence $\Phi_{Y}=U_{Y} \circ R$ where $U_{Y}$ is an involution defined near $p$. We do not say that $\Phi_{Y}$ is $R$-reversible, however, since $\operatorname{dim} \Sigma_{p}=2 n-1$. But we do have that $\operatorname{dim} F\left(U_{Y}\right)$ $=\operatorname{dim} F(R)=n$. This follows since $x \in F\left(U_{Y}\right)$ iff

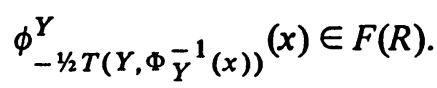

Set $\Psi_{Y}(w)=\Psi(Y, w)$. From (7.1) and (7.2), it follows that $\Psi_{Y}(W) \subset$ $F\left(U_{Y}\right)$ for each $Y \in O$.

A symmetric periodic orbit $\gamma$ of $X$ through $p \in F(R)$ is called 0-elementary on $\Sigma_{p}$ if there exists an $R$-invariant local transversal section $\Sigma_{p}$ on which $\Psi_{X}$ is transverse to $F(R) \cap \Sigma_{p}$. Equivalently, $\gamma$ is 0 -elementary if $F\left(U_{X}\right) 币 F(R)$ at $p$. This definition is independent of the particular section chosen, and does not apply to orbits which close up only after two or more iterates of the Poincaré map.

Proposition 7.3. Let $s \geqslant 1$ and suppose $X \in \Gamma_{R}^{s}(M)$ has a symmetric periodic orbit $\gamma$ through $p \in F(R)$.

(i) Then there exists a local transversal section $\Sigma_{p}$, a neighborhood 0 of $X$ in $\Gamma_{R}^{s}(M)$ and a dense, open subset $D_{s}$ of $O$ for which $Y \in D_{s}$ has only 0elementary orbits on $\Sigma_{p}$.

(ii) If $\gamma_{0}$ is a 0elementary closed orbit of $Y$ on $\Sigma_{p}$, then there exists $a$ smooth one-parameter family of symmetric periodic orbits of $Y$ containing $\gamma_{0}$.

Proof. The proof is similar to Abraham-Robbin [2, p. 109]. Choose 0 , $\Sigma_{p}$, and $W$ as above so that the associated semi-Poincare map is defined and $C^{s}$ on $W$. We show $\Psi$ is $C^{s}$ transverse to $F(R) \cap \Sigma_{p}=\Sigma_{R}$ if $s \geqslant 2$.

Suppose $\Psi(Y, y)=x \in \Sigma_{R}$. Let $\tau=T(Y, x)$ and pick any $g:[0, \tau / 2] \rightarrow \mathbf{R}$ $C^{\infty}$ and satisfying $g^{(k)}(0)=0=g^{(k)}(\tau / 2)$ for $0 \leqslant k \leqslant s$ and

$$
\int_{0}^{\tau / 2} g(s) d s=1
$$

Let $\dot{x} \in T_{x} \Sigma_{p}$. Define $\xi\left(\phi_{-s}(x)\right)=g(s) T_{x} \phi_{-s}(\dot{x})$ for $0 \leqslant s \leqslant \tau / 2$. Set $\xi\left(\phi_{s}(x)\right)$ $=-T R\left(\xi\left(\phi_{-s}(x)\right)\right)$ and note that $\xi$ is $C^{s} R$-reversible along $\phi_{s}(x)$ ( $\xi$ is $C^{s}$ at $x$ and $y$ by the conditions on the derivatives of $g$ ).

Extend $\xi$ to a $C^{s}$ vector field on $M$ by means of a partition of unity. Let $\eta=1 / 2(\xi-T R(\xi)) . \eta \in \Gamma_{R}^{s}(M)$ and $\eta=\xi$ on $\phi_{s}(x)$. Let $\phi_{t}^{\lambda}=$ flow of $Y+\lambda \eta$ where $\lambda$ is real. Then

$$
(d / d \lambda)\left(\phi_{\tau / 2}(y)\right)=\int_{0}^{\tau / 2} T \phi_{s}^{0} \circ \eta \circ \phi_{-s+\tau / 2}(y) d s=\dot{x} .
$$


Thus, $T_{(Y, y)} \Psi$ is surjective and (i) follows.

Now $\Psi_{Y}^{-1}\left(\Sigma_{R}\right)$ has codimension $n-1$ in $W$ and thus is a submanifold of dimension one. This submanifold generates the one-parameter family of closed orbits and thus proves (ii).

The proof for $s=1$ uses the indifference of $s$.

A symmetric periodic orbit is called $N$-elementary if the principal characteristic multipliers are $N$-elementary. As in the Hamiltonian case, not all symmetric periodic orbits can be made $N$-elementary. See Robinson [13] for a discussion of how this happens. Meyer and Palmore [9] have an example to show this phenomenon actually occurs; their example is a Hamiltonian vector field, but it is easily seen to be $R$-reversible also.

If $X$ has $\gamma$ as a 0 -elementary symmetric periodic orbit, we may choose an $R$-invariant neighborhood of $p \in F(R) \cap \gamma$ with coordinates as above. For any neighborhood $\bar{O}$ of $X$, the bundle $\bar{O} \times T N \rightarrow \bar{O} \times N$ admits $2 n$ global cross-sections defined by:

$$
\begin{array}{rlrl}
v_{i}(Y, x) & =\left(Y,\left(\partial / \partial x_{i}\right)(x)\right), & & i=1, \ldots, n, \\
v_{n+i}(Y, x) & =\left(Y,\left(\partial / \partial y_{i}\right)(x)\right), & i=1, \ldots, n-1, \\
\nu_{2 n}(Y, x) & =(Y, Y(x)) . & &
\end{array}
$$

For $\overline{0}$ small enough, we may assume:

(i) All orbits corresponding to fixed points of the Poincaré map are 0-elementary.

(ii) $\Psi: W \rightarrow \Sigma_{p}$ is defined and $C^{s}$.

(iii) The $2 n$ cross-sections above are pointwise linearly independent.

Now let $\pi: L_{R}(T N) \rightarrow N$ be the bundle given by

$$
\pi^{-1}(x)=\left\{f \in L\left(T_{x} N, T_{R x} N\right) \mid f \circ T R=T R \circ f^{-1}\right\} .
$$

Let $D_{R}=D_{R} \mathbf{R}^{2 n}$ be the set of $R$-reversible linear maps of $\mathbf{R}^{2 n}$. The cross-sections above give a map $\rho_{1}: \overline{0} \times L_{R}(T N) \rightarrow N \times D_{R}$. Define $\rho_{2}: \overline{0} \times W \rightarrow$ $\overline{0} \times L_{R}(T N)$ by

$$
\rho_{2}(X, w)=\left(Y, T_{x} \phi_{T(Y, x)}^{Y}(x)\right)
$$

where $x=\Phi_{Y}^{-1} \Psi(w)$.

Finally, define $\hat{\Psi}=\rho_{1} \circ \rho_{2}$. Note that $\hat{\Psi}$ actually takes values in $\Sigma_{p} \times J$ where $J=J\left(\partial / \partial y_{n}\right)$ is defined as in $\S 5 . J^{N}$ is the set of $R$-reversible linear maps that are not sub- $N$-elementary. A symmetric periodic orbit given by transversal intersection of $\hat{\Psi}$ with $J^{N}$ is called a bifurcation orbit.

Proposition 7.4. Let $s \geqslant 2, X \in \Gamma_{R}^{s}(M)$, and $\gamma$ a 0-elementary closed orbit of $X$. Then there exists an $R$-invariant section $\Sigma_{p}$ for $X$, a neighborhood 0 of $X$, and a dense, open subset $S^{N}$ of $O$ such that

(i) all but a finite number of the symmetric periodic orbits corresponding 
to fixed points of the Poincare map on $\Sigma_{p}$ are $N$-elementary.

(ii) The non- $N$-elementary symmetric periodic orbits are bifurcation orbits.

Proof. Choose $O$ as above and define $\rho: 0 \rightarrow C^{s}\left(W, \Sigma_{p} \times J\right)$ by ev० $\rho$ $=\hat{\Psi} . J^{N}$ is a finite union of submanifolds $J_{1}^{N}, \ldots, J_{k}^{N}$ of $J$ by Proposition 5.4. We show $\rho$ is $C^{s-1}$ transverse to $\Sigma_{R} \times J_{i}^{N}$ for each $i$, where $\Sigma_{R}=\Sigma_{p} \cap F(R)$.

Since $\Sigma_{R}$ has codimension $n-1$ in $\Sigma_{p}$ and each $J_{i}^{N}$ has codimension at least one in $J$, it follows that $\hat{\Psi}_{Y}^{-1}\left(\Sigma_{R} \times J^{N}\right)$ is a submanifold of dimension zero, or a finite set of points in $W$.

To see that $\rho$ is $C^{s-1}$ transverse to $\Sigma_{R} \times J_{i}^{N}$, first note that $\hat{\Psi} \pitchfork \Sigma_{R}$ since all closed orbits are 0 -elementary. The following lemma yields $\hat{\Psi} \Phi J_{i}^{N}$.

LEMMA 7.5. Let $Y \in \Gamma_{R}^{s}(M)$ have a symmetric periodic orbit $\gamma$ through $p \in F(R)$, and let $U$ be a neighborhood of $\gamma$ in $M$. Let per $\gamma=\tau$ and $\phi_{t}=$ flow of $Y$. Let $L=T_{p} \phi_{\tau} \in D_{R}\left(T_{p} M\right)$ and choose $A \in T_{L} D_{R}\left(T_{p} M\right)$ such that $A\left(Y_{p}\right)=0$. Then, if $s \geqslant 2$, there exists $\xi \in \Gamma_{R}^{s}(M)$ such that:

(i) $\left.\xi\right|_{\gamma}=0$,

(ii) $\left.\xi\right|_{M-U}=0$,

(iii) $\left.(\partial / \partial \lambda)\left(T_{p} \phi_{\tau}^{\lambda}\right)\right|_{\lambda=0}=A$ where $\phi_{t}^{\lambda}=$ flow of $Y+\lambda \xi$.

Proof. The proof is a modification of the tangent perturbation lemma in Abraham-Robbin [2, p. 111]. They construct $\eta \in \Gamma^{s}(M)$ satisfying (i), (ii), and (iii). Let $\xi=1 / 2(\eta-T R(\eta))$. Then $\xi \in \Gamma_{R}^{s}(M)$ and $\xi$ satisfies (i) and (ii). To see (iii) also holds, note that, in the proof in [2],

and

$$
H(x, T R(\dot{x}))=T^{2}\left(R \phi_{-\tau}\right) H\left(x, T_{x} \phi_{-\tau}(\dot{x})\right)
$$

$$
B(\dot{y})=-T^{2} R \circ B \circ T R(\dot{y})
$$

The lemma then follows immediately. Finally, the proof of the proposition for $s=2$ uses the indifference of $s$.

We now combine the two previous local lemmas with a method of Peixoto and Robinson to prove the Kupka-Smale theorem for $R$-reversible vector fields.

$X \in \Gamma_{R}^{S}(M)$ is called $N$-generic (for orbits of period $\leqslant \tau$ ) if

(i) $X \in H^{s}(N)$,

(ii) all symmetric periodic orbits (of period $\leqslant \tau$ ) have local transversal sections on which the conclusions of Proposition 7.4 hold, and

(iii) all nonsymmetric periodic orbits (of period $\leqslant \tau$ ) are hyperbolic.

Let $K^{s}(N)\left(K^{s}(N, \tau)\right)$ be the set of all $C^{s} R$-reversible vector fields that are $N$-generic (for orbits of period $\leqslant \tau$ ). Set $K^{s}=\bigcap_{N=1}^{\infty} K^{s}(N)$.

THEOREM 7.6. If $M$ is compact and $s \geqslant 2, K^{s}(N)$ is residual in $\Gamma_{R}^{s}(M)$. Thus, $K^{s}$ is also residual. 
Proof. Robinson's proof for Hamiltonian vector fields goes over exactly for the $R$-reversible case. See [13]. Use the usual Kupka-Smale theorem for the nonsymmetric periodic orbits. We omit the details.

VIII. Liapounov's theorem. In this section, we show that another qualitative property of Hamiltonian vector fields carries over to the $R$-reversible case. The classical Liapounov theorem states that, if an analytic Hamiltonian vector field has a purely imaginary characteristic exponent $i \lambda$ at a singular point $p$, then, subject to certain resonancy restrictions, there exists an analytic one-parameter family of nested closed orbits of the vector field about $p$. Moreover, the periods of the closed orbits tend to $2 \pi /|\lambda|$ as the initial conditions converge to $p$. For a proof, see [15] . [1, Appendix C] contains a generalization to the $C^{1}$ case. Recently, some of the resonance conditions have been relaxed. See, for instance, [19].

Let $X$ be a $C^{2} R$-reversible vector field in a neighborhood of a symmetric singular point $p$. Let $\lambda=\lambda_{1}, \ldots, \lambda_{n}$ be the principal characteristic exponents of $X$ at $p$ and suppose $\lambda$ is purely imaginary.

THEOREM 8.1. In the above situation, if no other principal eigenvalue is equal to $i \lambda n$ for any integer $n$, then there exists a $C^{2}$, two-dimensional, invariant manifold $M^{\lambda}$ containing $p$ with the property that $M^{\lambda}$ consists of a nested, oneparameter family of symmetric periodic orbits. Moreover, the periods of the closed orbits tend to $2 \pi /|\lambda|$ as the initial conditions tend to $p$.

REMARK. Note that the resonancy restrictions are met if $p$ is an elementary symmetric singularity of $X$. Of course, the theorem is not true if $p$ is a nonsymmetric singular point of $X . M^{\lambda}$ is called a Liapounov subcenter manifold.

Proof. By $R$-reversibility, if an orbit of $X$ has two distinct intersections with $F(R)$, the orbit is symmetric periodic. We show there exists a one-dimensional submanifold $S$ of $F(R)$ containing $p$ with the property that each point of $S$ returns to $F(R)$ after time approximately $\pi /|\lambda|$.

Take $C^{\infty}$ coordinates $\left(x_{i}, y_{i}\right), i=1, \ldots, n$, on a neighborhood $U$ of $p$ with $y_{i}=0$ defining $F(R) \cap U$ and $x_{i}(p)=0$. Let $\phi_{t}$ be the flow of $X$ and $\pi_{1}$ the projection from $U$ onto the $y_{i}$-subspace. By the results of $\S 5$, we may assume that $\partial / \partial x_{1}$ and $\partial / \partial y_{1}$ span the -1 eigenspace of $T \phi_{\pi /|\lambda|}(p)$.

Now let $\tau>\pi /|\lambda|$. Choose an open set $W \subset F(R) \cap U$ satisfying $\phi_{t}(W) \subset U$ for all $t$ in the interval $[0, \tau]$. Define $F: W \times[0, \tau] \rightarrow \mathbf{R}^{n}$ by $F(x, t)=$ $\pi_{1} \circ \phi_{t}(x)$. Thus the orbit through $x$ is symmetric periodic if there exists $t>0$ with $F(x, t)=0$.

Define $G: W \times[0, \tau] \rightarrow \mathrm{R}^{n-1}$ by $G(x, t)=\pi_{0} \circ F(x, t)$ where $\pi_{0}$ is the projection on the last $n-1$ coordinates. One checks easily that $T G(p, \pi /|\lambda|)$ has rank $n-1$. Hence $G^{-1}(0)$ is a two-dimensional submanifold of $W \times[0, \tau]$ locally at $(p, \pi /|\lambda|)$. Note that $G^{-1}(0)$ contains the curve $t \rightarrow(p, t)$. 
Now define $H: G^{-1}(0) \rightarrow \mathrm{R}$ by $H=\pi_{2} \circ F$ where $\pi_{2}$ is the projection on the $y_{1}$-axis in $R^{n}$. An easy computation shows that $(p, \pi /|\lambda|)$ is a nondegenerate critical point of index one for $H$. Hence, using the Morse lemma, $H^{-1}(0)$ consists locally of two one-dimensional submanifolds intersecting transversally at $(p, \pi /|\lambda|)$. One of these submanifolds is the curve $t \rightarrow(p, t)$. The other consists of points of the form $(x, t(x))$ satisfying $F(x, t(x))=0$. These points thus define the submanifold $S$ of $F(R)$.

The second part of the theorem follows from the fact that $(p, \pi /|\lambda|) \in H^{-1}(0)$. This completes the proof.

IX. Homoclinic orbit theorems. Throughout this section, let $p$ be a symmetric hyperbolic critical point of the $R$-reversible vector field $X$. Let

$$
W^{s}(p)=\left\{x \in M \mid \phi_{t}(x) \rightarrow p, t>0\right\} .
$$

$W^{s}(p)$ is called the stable manifold for $X$ at $p$. We also define the unstable manifold for $X$ at $p$ to be the stable manifold for $-X$ at $p$. Let $W^{u}(p)$ denote the unstable manifold. It is well known that both $W^{s}(p)$ and $W^{u}(p)$ are immersed invariant submanifolds of $M$ [2]. By 5.2, $\operatorname{dim} W^{s}(p)=\operatorname{dim} W^{u}(p)=n$, and by $R$-reversibility, we have $R\left(W^{s}(p)\right)=W^{u}(p)$.

We also assume that $W^{s}(p)$ has a point of transversal intersection $q \neq p$ with $F(R)$. By symmetry, $q \in W^{u}(p)$, and by invariance of the stable and unstable manifolds, it follows that the orbit of $q, \phi_{t}(q)$, lies in $W^{s}(p) \cap W^{u}(p)$. Such an orbit is called a symmetric homoclinic orbit. We remark that, since $W^{s}(p)$ is transverse to $F(R)$ at $q$, it follows that the symmetric homoclinic orbit through $q$ cannot be perturbed away. This, of course, is quite unlike the situation for ordinary vector fields. In this case, the sum of the dimensions of $W^{s}(p)$ and $W^{u}(p)$ is exactly the dimension of the manifold. Hence $W^{s}(p)$ and $W^{u}(p)$ cannot intersect transversely along a one-dimensional homoclinic orbit and may thus be perturbed away.

The following theorem states that homoclinic orbits are "almost" periodic orbits in that they are the limits of one-parameter families of symmetric closed orbits. A similar theorem is known in the Hamiltonian case only for systems with two degrees of freedom [21].

THEOREM 9.1. Let $p$ be a symmetric hyperbolic zero of $X$, and let $q \neq p$ be a point of transversal intersection of $W^{s}(p)$ and $F(R)$. Then the homoclinic orbit through $q$ is the limit of a one-parameter family of symmetric closed orbits whose periods tend to $\infty$.

Proof. Let $V$ be a disk in $W^{u}(p)$ containing $p$. Since $V$ is transverse to $F(R)$ at $p$, there is an $\epsilon>0$ such that any disk $C^{1}-\epsilon$ close to $V$ in $M$ also has a unique point of transversal intersection with $F(R)$ near $p$.

Now let $N$ be a neighborhood of $q$ in $F(R)$. By the $\lambda$-lemma [22], there is 
a $\tau>0$ such that, for any $t>\tau$, there exists a disk $N_{t} \subset N$ such that $\phi_{t}\left(N_{t}\right)$ is $C^{1}-\epsilon$ close to $V$. Hence $\phi_{t}\left(N_{t}\right)$ intersects $F(R)$ transversally at a point $x_{t}$. Since $N_{t}$ varies continuously with $t$, it follows that $x_{t}$ is a curve in $F(R)$ approaching $p$ as $t$ approaches $\infty$. Finally, $\phi_{t}\left(x_{t}\right) \in F(R)$, and $\phi_{-t}\left(x_{t}\right) \rightarrow q$, so the orbits through $x_{t}$ are symmetric periodic and tend to the orbit of $q$.

Appendix. Sections with boundary. The purpose of this appendix is to give the basic definitions and properties of sections with boundary for vector fields on a manifold. Most of this material can be found in Birkhoff's paper [4]. What we add here is a criterion for the stability of such sections under perturbations of the vector field.

Throughout, we assume $M$ is an orientable $C^{\infty}$ manifold of dimension $n$ and $X$ is a $C^{2}$ vector field on $M$ with flow $F_{t}$. A section with boundary for $X$ on $M$ is a pair $(\Sigma, \partial \Sigma)$ where $\Sigma$ is an orientable submanifold of codimension one in $M$ and $\partial \Sigma$ is the boundary of $\Sigma$. $(\Sigma, \partial \Sigma)$ has the additional properties that:

(i) $\partial \Sigma$ is an invariant manifold for $X$,

(ii) $X$ is transverse to $\Sigma-\partial \Sigma$,

(iii) there exists $T \in \mathrm{R}, T>0$, such that if $x \in \Sigma-\partial \Sigma$, there exist $t, s \in$ $\mathbf{R}, 0<t<T,-T<s<0$, with $F_{t}(x) \in \Sigma$ and $F_{s}(x) \in \Sigma$.

Sections without boundary have often been used to reduce the study of qualitative properties of a vector field to the study of the corresponding properties of the associated first return diffeomorphism. Here we show that, with minor restrictions, a similar reduction is possible in the boundary case. For the basic definitions and properties of sections without boundary, see [16].

If $X$ admits a section with boundary $(\Sigma, \partial \Sigma)$, there is an associated first return map $L: \Sigma-\partial \Sigma \rightarrow \Sigma-\partial \Sigma$ defined by $L(x)=F_{T(x)} x$ where $T(x)$ is the first positive real number for which $F_{T(x)} x \in \Sigma$. T is called the time of first return map. $L$ is easily a diffeomorphism of $\Sigma-\partial \Sigma$ into itself.

To extend $L$ over $\partial \Sigma$ differentiably, we need the following notions. Let $T \Sigma$ denote the tangent bundle of $\Sigma$ considered as an embedded $(2 n-2)$-dimensional submanifold of $T M$. We say $\Sigma$ is regularly bounded if $X$ is transverse to $T \Sigma$. Here we regard $X$ as a $C^{2}$ map $M \rightarrow T M$. Note that $X(p) \in T \Sigma$ iff $p \in \partial \Sigma$.

Proposition A.1. Let $X \in \Gamma^{2}(M)$ admit a regularly bounded section with boundary $(\Sigma, \partial \Sigma)$. Then the first return map $L$ extends to a $C^{1}$ diffeomorphism of $\Sigma$.

PROOF. It suffices to show that the time of first return map $T$ extends over $\partial \Sigma$ in a $C^{1}$ manner.

To see this, take a neighborhood $W$ of $\partial \Sigma$ in $M$ with $W$ diffeomorphic to $\partial \Sigma \times$ $\mathbf{R}^{\mathbf{2}}$. This diffeomorphism can be constructed using the orientability of $\Sigma$ and a $C^{\mathbf{2}}$ collaring of $\partial \Sigma$ in $\Sigma$. Let $x, z$ be global coordinates on $W$ with $z=0, x \geqslant 0$ defining $\Sigma \cap W$. 
Let $V$ be an open neighborhood of $\partial \Sigma$ in $\Sigma \cap W$ with $L(p) \in \Sigma \cap W$ for all $p \in \bar{V}-\partial \Sigma$.

Define

$$
g(p, t)= \begin{cases}z \circ F_{t}(p) / x(p), & p \in \bar{V}-\partial \Sigma \\ (\partial / \partial x)\left(z \circ F_{t}\right)(p), & p \in \partial \Sigma\end{cases}
$$

$g$ is defined and $C^{1}$ is a neighborhood $N$ of $\{(p, T(p)) \mid p \in \bar{V}-\partial \Sigma\}$. Note that $g(p, T(p))=0$ for $p \in \bar{V}-\partial \Sigma$. Thus $T$ extends over $\partial \Sigma$ providing we can solve $g(p, t)=0$ for $t$.

But this can be done if $(\partial g / \partial t)(p, t) \neq 0$ for $p \in \partial \Sigma$. An easy computation then shows that this condition is a coordinate definition of regular boundedness.

One method of constructing sections with boundary is to suspend a given diffeomorphism. More precisely, let $L: \Sigma \rightarrow \Sigma$ be a diffeomorphism. Suppose $L \mid \partial \Sigma$ is the time-one map of some flow $\phi_{t}$ on $\partial \Sigma$ (this is a real restriction, since not all diffeomorphisms can be so embedded in a flow). Let $M$ be the quotient space of $\Sigma \times \mathbf{R}$ obtained by identifying $(x, t)$ with $(L(x), t-1)$ and $(y, s)$ with $\left(\phi_{t}(y), s-t\right)$ when $y \in \partial \Sigma$. $M$ is easily seen to be a manifold without boundary of dimension $1+\operatorname{dim} \Sigma$.

The flow generated by the constant vector field $(0,1)$ on $\Sigma \times \mathbf{R}$ then projects to a new flow on $M$ having an embedded copy of $(\Sigma, \partial \Sigma)$ as a section with boundary and $L$ as the first return map. This flow is called the suspension of $L$.

In practice, the existence of sections with boundary is often difficult to verify. See Birkhoff [4] for a discussion of this problem for Hamiltonian systems with two degrees of freedom. For higher dimensional systems, existence of sections with boundary seems rare. One would first need a compact $(n-2)$-dimensional invariant manifold for the flow, which probably does not exist generically. Nevertheless, with suitable hypotheses, the existence of sections with boundary is an open condition in $\Gamma^{s}(M)$.

THEOREM A.2. Let $X \in \Gamma^{2}(M)$ admit a regularly bounded section with boundary $(\Sigma, \partial \Sigma)$. Suppose there exists a neighborhood $N_{0}$ of $X$ in $\Gamma^{2}(M)$ and a continuous map $\Phi: N_{0} \rightarrow \operatorname{Diff}(M)$ such that if $Y \in N_{0}, \Phi(Y)(\partial \Sigma)$ is an invariant manifold for $Y$. Then there exists a possibly smaller neighborhood $N$ of $X$ such that all $Y \in N$ admit regularly bounded sections with boundary.

Proof. First choose neighborhoods $W$ and $V$ of $\partial \Sigma$ in $M$ and $\Sigma$ respectively as in Proposition A.1. Let $x, z$ be global coordinates on $W$ as before. Define $h_{Y}: \bar{V} \times \mathbf{R} \rightarrow M$ by

$$
h_{Y}(p, t)=f_{Y}^{-1} \circ F_{t}^{Y} \circ f_{Y}
$$

where $Y \in N_{0}, f_{Y}=\Phi(Y)$, and $F_{t}^{Y}=$ flow of $Y$. Also define 


$$
\psi_{Y}(p, t)= \begin{cases}z \circ h_{Y}(p, t) / x(p), & p \in \bar{V}-\partial \Sigma, \\ (\partial / \partial x)\left(z \circ h_{Y}\right)(p, t), & p \in \partial \Sigma .\end{cases}
$$

As before, there exists a neighborhood $U$ of $\{(p, T(p)) \mid p \in \bar{V}\}$ in $\bar{V} \times \mathbf{R}$ such that $\psi_{Y}: U \rightarrow \mathrm{R}$ is defined and $C^{1}$. Thus we get a continuous map $\Psi: N_{0} \rightarrow$ $C^{1}(U, \mathrm{R})$ given by

$$
e v \circ \Psi(Y, p, t)=\psi_{Y}(p, t)
$$

Continuity arguments then give a neighborhood $N_{1}$ of $X$ such that each $Y \in N_{1}$ has $f_{Y}^{-1}(\bar{V})$ as a local section with boundary. $f_{Y}^{-1}(\bar{V})$ is regularly bounded via the openness of transversal intersection.

To complete the proof, choose a neighborhood $V_{1}$ of $\partial \Sigma$ in $\Sigma$ such that $\partial \Sigma \subset \bar{V}_{1} \subset V$. Let $V_{1}^{c}$ be the complement of $V_{1}$ in $\Sigma$. Let $\epsilon>0$. There exists an open neighborhood $N_{2}$ of $X$ such that each $Y \in N_{2}$ has the property that, for any $p \in V_{1}^{c}$, the integral curve of $Y$ through $f_{Y}(p)$ returns to $f_{Y}(\Sigma)$ at least once before time $T(p)+\epsilon$. This follows from the transversality of the flow of $X$ on $V_{1}^{c}$ and the compactness of $V_{1}^{c}$.

Let $N=N_{1} \cap N_{2}$. Then each $Y \in N$ thus has $f_{Y}(\Sigma)$ as a regularly bounded section with boundary. This completes the proof.

\section{REFERENCES}

1. R. Abraham and J. E. Marsden, Foundations of mechanics. A mathematical exposition of classical mechanics with an introduction to the qualitative theory of dynamical systems and applications to the three-body problem, Benjamin, New York and Amsterdam, 1967. MR 36 \#3527.

2. R. Abraham and J. Robbin, Transversal mappings and flows, Benjamin, New York, 1967. MR 39 \#2181.

3. G. Birkhoff, The restricted problem of three bodies, Rend. Circ. Mat. Palermo 39 (1915), 265-334.

4. Dynamical systems with two degrees of freedom, Trans. Amer. Math. Soc. 18 (1917), 199-300.

5. M. Braun, Particle motions in a magnetic field, J. Differential Equations 8 (1970), 294-332. MR 41 \#9496.

6. R. De Vogelaere, On the structure of symmetric periodic solutions of conservative systems, with applications, Contributions to the Theory of Nonlinear Oscillations, rol. IV, Ann. of Math. Studies, no. 41, Princeton Univ. Press, Princeton, N. J., 1958, pp. 53-84. MR 21 \#174.

7. Surface de section dans le problème de Störmer, Acad. Roy. Belg. Bull. Cl. Sci. (5) 40 (1954), 705-714. MR 16, 312.

8. C. Graef Fernández, Periodic orbits of the primary cosmic radiation, Bol. Soc. Mat. Mexicana 1 (1944), no. 3, 1-31. (Spanish) MR 6, 75.

9. K. R. Meyer and J. Palmore, A generic phenomenon in conservative Hamiltonian systems, Proc. Sympos. Pure Math., vol. 14, Amer. Math. Soc., Providence, R. I., 1970, pp. 185-189. MR 42 \#3811.

10. D. Montgomery and L. Zippin, Topological transformation groups, Interscience, New York, 1955. MR 17, 383.

11. R. S. Palais, Equivalence of nearby differentiable actions of a compact group, Bull. Amer. Math. Soc. 67 (1961), 362-364. MR 24 \#A185.

12. M. M. Peixoto, On an approximation theorem of Kupka and Smale, J. Differential Equations 3 (1967), 214-227. MR 35 \#499. 
13. R. C. Robinson, A global approximation theorem for Hamiltonian systems, Proc. Sympos. Pure Math., vol. 14, Amer. Math. Soc., Providence, R. I., 1970, pp. 233-243. MR 42 \#3812.

14. - Generic properties of conservative systems, Amer. J. Math. 92 (1970), 562-603. MR 42 \#8517.

15. C. Siegel and J. Moser, Lectures on celestial mechanics, Springer-Verlag, New York, 1971.

16. S. Smale, Stable manifolds for differential equations and diffeomorphisms, Ann. Scuola Norm. Sup. Pisa (3) 17 (1963), 97-116. MR 29 \#2818b.

17. Topology and mechanics. II. The planar n-body problem, Invent. Math. 11 (1970), 45-64. MR 47 \#671.

18. C. Störmer, Sur les trajectoires des corpuscules électrisés dans l'espace sous l'action du magnétisme terrestre avec application aux aurores boréales, Arch. Sci. Phys. Nat. 24 (1907), 113-158.

19. J. Roels, An extension to resonant cases of Liapunov's theorem concerning the periodic solutions near a Hamiltonian equilibrium, J. Differential Equations 9 (1971), 300324. MR $42 \# 8036$.

20. M. S. Vallarta, An outline of the allowed cone of cosmic radiation, Univ. of Toronto Press, Toronto, 1938.

21. J. Henrard, Proof of a conjecture of Stromgren, Celestial Mechanics 7 (1973), $449-457$.

22. J. Palis, On Morse-Smale dynamical systems, Topology 8 (1968), 385-404. MR 39 \#7620.

DEPARTMENT OF MATHEMATICS, NORTHWESTERN UNIVERSITY, EVANSTON, ILLINOIS 60201 